\title{
Estimación del impacto económico de la equinococosis quística en Chile y análisis de las posibles causas que han dificultado su erradicación
}

\author{
JUAN VENEGAS ${ }^{1, a}$, SANDRA ESPINOZA²,b, GITTITH SÁNCHEZ ${ }^{1, a}$
}

\section{Estimation of costs caused by cystic echinococcosis}

Background: Hydatid disease or cystic echinococcosis, caused by the parasite Echinococcus granulosus, has a worldwide distribution, affecting people of working age and can cause high levels of morbidity and even death. Aim: To estimate the economic impact at the human and animal level caused by the disease in Chile. Material and Methods: We analyzed information about the disease obtained from reports and publications emanated from the Chilean Ministry of Health, United Nations Food and Agriculture Organization, the U.S. National Institute of Statistics and the National Agricultural Service. Animal derived costs were estimated evaluating the expenses for pharmacological treatment of infected dogs and animal production losses derived from confiscations and reductions in meat production. Results: The total number of patients who underwent surgery to remove a hydatid cyst in Chile during 2012, was estimated as 767 individuals. The annual costs derived only from surgical treatment, were estimated in USD 2.46 million. Summing the costs of sick leaves and loss of productivity, the costs at the human level ascended to USD 3.13 million. Considering human and animal costs, the annual economic burden of the disease was estimated in USD 14.35 million. Conclusions: The Analysis of the regional distribution of human and animal hydatidosis, suggests a significant environmental contamination with parasite eggs in high incidence regions such as Aysén, Araucanía, BioBio and Coquimbo. The efficiency of control programs for the disease would be greatly improved if the causes for these regional contaminations are elucidated.

(Rev Med Chile 2014; 142: 1023-1033)

Key words: Echinococcosis; Echinococcus granulosus, economics; Financial management.

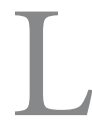
a equinococosis quística o hidatidosis es una enfermedad parasitaria de amplia distribución mundial producida por quistes del estadio larval del platelminto Echinococcus granulosus ${ }^{1}$. Esta enfermedad es la principal causa de hospitalización por enfermedad parasitaria en el país, puede producir altos grados de morbilidad a personas que en su mayoría están en plena edad productiva ${ }^{2,3,4,5}$. Los quistes se localizan preferentemente en el hígado y el tratamiento de elección

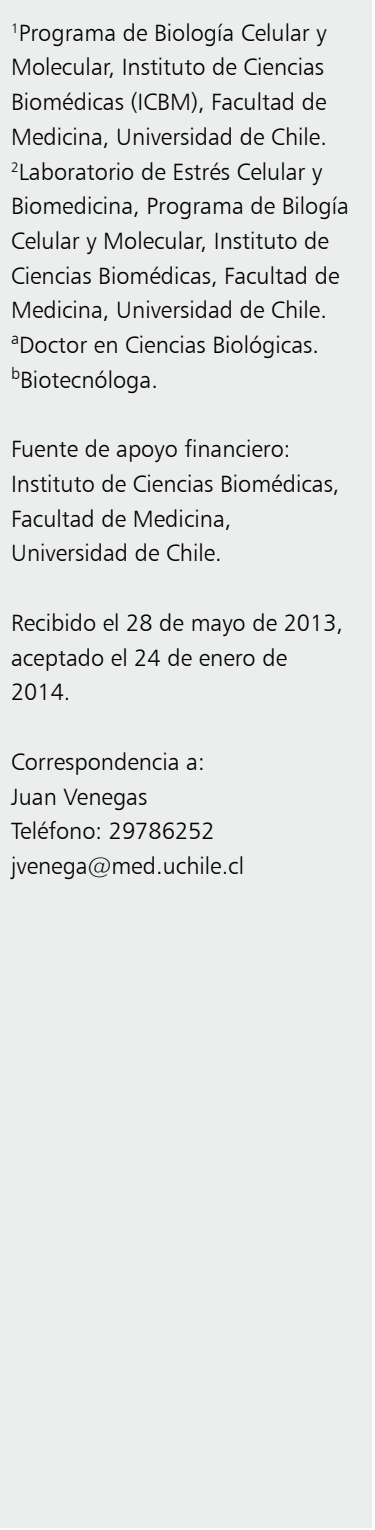

es quirúrgico, lo que produce altos costos económicos y sociales ${ }^{1}$.

En Chile, la hidatidosis es una enfermedad de notificación obligatoria (ENO), cuya incidencia a nivel nacional es de 1,48 casos por cada 100.000 habitantes ${ }^{6}$. Sin embargo, varios autores han propuesto que hay una evidente subnotificación de esta enfermedad, debido a que los datos de egresos hospitalarios generalmente triplican las cifras $\mathrm{ENO}^{2,3,4,5,7}$. 
Las estimaciones del impacto económico de la equinococosis quística tanto a nivel humano como animal, en nuestro país son escasas. Dentro de los pocos estudios se encuentra una estimación del costo económico producido por el tratamiento quirúrgico de la hidatidosis, en el cual se analizaron 16 pacientes del Hospital San Juan de Dios, de Santiago ${ }^{8}$. A nivel animal, los primeros estudios realizados en Chile del impacto económico de esta enfermedad, se evaluó el efecto de los decomisos de hígados y riñones por detección post-mórtem de quistes hidatídicos en mataderos en bovinos, caprinos, equinos, porcinos y ovinos ${ }^{9}$. Aquí, no se consideró otras variables, tales como las pérdidas producidas en la producción de carnes. En un estudio posterior, en el cual sólo se consideró a bovinos y ovinos, se estimó una pérdida total en Chile entre USD 7 y 11,2 millones $^{10}$.

El presente estudio tiene como objetivo estimar el impacto económico a nivel humano y animal, que produce la equinococosis quística en nuestro país. Se realizó a partir de los datos actuales disponibles de literatura y de informes emanados por los organismos competentes. Se discuten las posibles causas que han dificultado la erradicación de la hidatidosis en Chile.

\section{Método}

Se realizó una investigación sistemática cualitativa que resume y analiza la información disponible en publicaciones e informes emanados por los organismos estatales competentes: Ministerio de Salud (Minsal), Organización de las Naciones Unidas para la Agricultura y la Alimentación Oficina Regional para América Latina y el Caribe (FAO/ RLC), Instituto Nacional de Estadísticas (INE), Servicio Agrario Ganadero (SAG), Organización Panamericana de la Salud-Organización mundial de la Salud (OPS-OMS), a partir de la cual, se realizaron todas las estimaciones mostradas en el presente artículo.

\section{Estimación del costo del tratamiento quirúrgico de la hidatidosis}

Se realizó basándose en los procedimientos descritos en Lorca et al., $2003^{8}$. Así, se consideraron 17 días promedio de hospitalización, una única intervención quirúrgica, y los exámenes descritos en la Tabla 2. Los costos público y privado de cada uno de estos exámenes, así como todo el procedimiento para realizar estas estimaciones, se describen en la Tabla 2.

\section{Resultados}

\section{Incidencia de hidatidosis a nivel regional y nacional}

En la Tabla 1, se muestra una estimación de la incidencia de la hidatidosis en Chile en el período 2001-2005 publicados por Cortés y Valle ${ }^{4}$. Esta estimación está basada en los datos de enfermedades de notificación obligatoria (ENO) y datos de egresos hospitalarios del Minsal, en los cuales se consideraron personas con diagnóstico según CIE- $10^{12}$ correspondientes a diferentes tipos de infecciones con E. granulosus (B67.0 a B67.4), con E. multilocularis (B67.5 a B67.7) y equinococosis no especificada (B67.8 y B67.9). Como se puede observar, en el período 2001-2005, en todas las regiones, la incidencia de hidatidosis según datos ENO, fue inferior a la incidencia estimada a partir de datos de egresos hospitalarios (Tabla 1). A nivel nacional, la incidencia ENO, en este período, era 2,7 veces inferior a la incidencia de egresos hospitalarios.

Para hacer una estimación del número total de personas sometidas a cirugía el año 2012, del cual no se dispone de los datos de egresos hospitalarios, se consideró el promedio del número total de casos ENO indicados por el Minsal para los años $2010^{13}$, $2011^{6}$ y $2012^{6}$, el cual da 255,7 multiplicado por 2,7 con lo cual se obtuvo una cifra estimada de 690 personas sometidas a cirugía.

Basados en que un diez por ciento de los pacientes que sufren la hidatidosis son tratados farmacológicamente, debido a que no pueden ser operados ${ }^{1}$, se asumió que los 690 pacientes corresponden a $90 \%$, y por lo tanto, $10 \%$ restante corresponde a 77 personas, por lo que la incidencia total estimada para el año 2012, fue de 767 personas.

$\mathrm{Al}$ analizar las incidencias en base sólo a datos ENO (Tabla 1), en el año 2012, la región con mayor incidencia fue Aysén, seguido por Los Ríos, Magallanes, Los Lagos, Biobío y La Araucanía.

\section{Estimación del costo económico producido por el tratamiento quirúrgico de la hidatidosis humana de acuerdo a costos en el sector público $y$ privado}

El procedimiento para estas estimaciones se describe en Métodos y la Tabla 2, están basados en 
Tabla 1. Incidencia y número de casos de hidatidosis humana por regiones, según casos notificados o egresos hospitalarios

\begin{tabular}{|c|c|c|c|c|c|c|c|c|c|c|}
\hline \multirow[b]{4}{*}{ Región } & \multirow[b]{4}{*}{ Nombre } & \multicolumn{8}{|c|}{ Años } & \multirow[b]{4}{*}{ Incid } \\
\hline & & \multirow{3}{*}{$\begin{array}{c}\text { 1996a }^{\mathrm{a}} \\
\text { Egresos } \\
\text { Incid.e }^{\text {e }}\end{array}$} & \multicolumn{2}{|c|}{$2001-2005^{b}$} & \multirow{2}{*}{\multicolumn{2}{|c|}{$\begin{array}{l}2010^{c} \\
\text { ENO }\end{array}$}} & \multirow{2}{*}{\multicolumn{2}{|c|}{$\begin{array}{l}2011^{d} \\
\text { ENO }\end{array}$}} & \multirow{3}{*}{$\begin{array}{c}2012^{d} \\
\text { ENO } \\
\text { Casos }\end{array}$} & \\
\hline & & & ENO & Egresos & & & & & & \\
\hline & & & Incid. & Incid. & Casos & Incid. & Casos & Incid. & & \\
\hline$X V$ & Arica y Parinacota & & & & 0 & 0,00 & 1 & 0,55 & 1 & 0,55 \\
\hline I & Tarapacá & 0,8 & 0,0 & 1 & 0 & 0,00 & 1 & 0,31 & 0 & 0,00 \\
\hline ॥ & Antofagasta & 2,9 & 0,0 & 1 & 0 & 0,00 & 0 & 0,00 & 4 & 0,68 \\
\hline III & Atacama & 5,1 & 0,5 & 2 & 0 & 0,00 & 4 & 1,42 & 0 & 0,00 \\
\hline IV & Coquimbo & 9,7 & 5,0 & 10,5 & 22 & 3,06 & 20 & 2,74 & 14 & 1,89 \\
\hline V & Valparaíso & 4,6 & 0,3 & 2 & 2 & 0,11 & 4 & 0,23 & 4 & 0,22 \\
\hline M & Metropolitana & 5,2 & 0,5 & 2 & 23 & 0,33 & 17 & 0,24 & 30 & 0,43 \\
\hline $\mathrm{VI}$ & O’Higgins & 5,0 & 1,0 & 3,8 & 7 & 0,79 & 12 & 1,35 & 8 & 0,89 \\
\hline VII & Maule & 12 & 4,6 & 9,8 & 12 & 1,19 & 15 & 1,48 & 16 & 1,56 \\
\hline VIII & Biobío & 8 & 4,4 & 9 & 70 & 3,44 & 69 & 3,37 & 69 & 3,35 \\
\hline IX & Araucanía & 39 & 4,0 & 28,5 & 34 & 3,50 & 45 & 4,60 & 31 & 3,14 \\
\hline XIV & Los Ríos & 13 & 4,0 & 9,9 & 13 & 3,42 & 21 & 5,52 & 28 & 7,34 \\
\hline$x$ & Los Lagos & & ND & ND & 4 & 0,48 & 5 & 0,59 & 32 & 3,73 \\
\hline$X I$ & Aysén & 80 & 38 & 44 & 44 & 41,97 & 38 & 35,89 & 28 & 26,20 \\
\hline XII & Magallanes & 33 & 9 & 16,5 & 9 & 5,67 & 4 & 2,51 & 6 & 3,76 \\
\hline Chile & & 9,0 & 2,2 & 6 & 240 & 1,40 & 256 & 1,48 & 271 & 1,56 \\
\hline
\end{tabular}

aMinisterio de Salud. Objetivos Sanitarios para Chile 2000-2010. Ministerio de Salud (Minsal), Departamento de Epidemiología, Primera edición, octubre de 2002. 'bestos datos corresponden a los publicados por Cortés y Valle ${ }^{4}$ en los cuales tanto para casos notificados como para los egresos hospitalarios, se consideraron personas con diagnóstico según $\mathrm{CIE}-10^{12}$ correspondientes a E. granulosus (B67.0 a B67.4), E. multilocularis (B67.5 a B67.7) y equinococosis no especificada (B67.8 y B67.9). cHidatidosis (CIE 10: B67). Situación epidemiológica. Semanas 1 a 52 año 2011 (\&). Departamento de Epidemiología. División de Planificación Sanitaria, Ministerio de Salud de Chile ${ }^{13}$. 'Hidatidosis (CIE 10: B67). Situación epidemiológica, semanas 1 a 52, año 2012 (01/01/2012 al 29/12//2012). Atlas de zoonosis y enfermedades transmitidas por vectores, año 2012. Departamento de Epidemiología. División de Planificación Sanitaria-Ministerio de Salud de Chile 6 . Incidencia = número de casos por cada cien mil habitantes $(1 / 100.000)$

el trabajo de Lorca et al., (2003) $)^{8}$. Multiplicando cada valor unitario por el número de exámenes promedio, el costo del tratamiento quirúrgico con todos sus exámenes y procedimientos en el sistema público y privado dio un valor estimado de $\$ 1.340 .049$ y 3.691 .881 equivalentes a USD 2.678 y USD 7.379 (según precio del dólar 500,3 al 4 de octubre de 2013).

\section{Estimación del costo producido por licencias} médicas y déficit de producción por días no trabajados

La estimación del costo por licencias médicas, se basó en un estudio publicado previamente ${ }^{8}$, considerando el sueldo mínimo imponible (\$210.000), que dio un valor por día de \$7.000, multiplicado por el número de días promedios de hospitalización ${ }^{17}$, se obtuvo \$119.000 (Tabla 2).

El costo por déficit de producción, considerando un PIB per cápita del año $2012^{16}$ de USD 15.424, dividido por 365 días, nos da USD 42,3 equivalentes a $\$ 21.175$ pesos (de acuerdo al precio del dólar indicado anteriormente). Multiplicado por los 17 días no trabajados, nos da un valor de \$359.982 (Tabla 2).

Estimación del costo total producido por tratamiento quirúrgico para extirpar un quiste hidatídico, licencias y pérdida de producción, por cada paciente

Sumando, los costos por tratamiento quirúrgico (\$1.340.049) más costo por licencias médicas 
Tabla 2. Estimación del costo por paciente, producido por el tratamiento quirúrgico para extirpar un quiste hidatídico hepático sin complicaciones

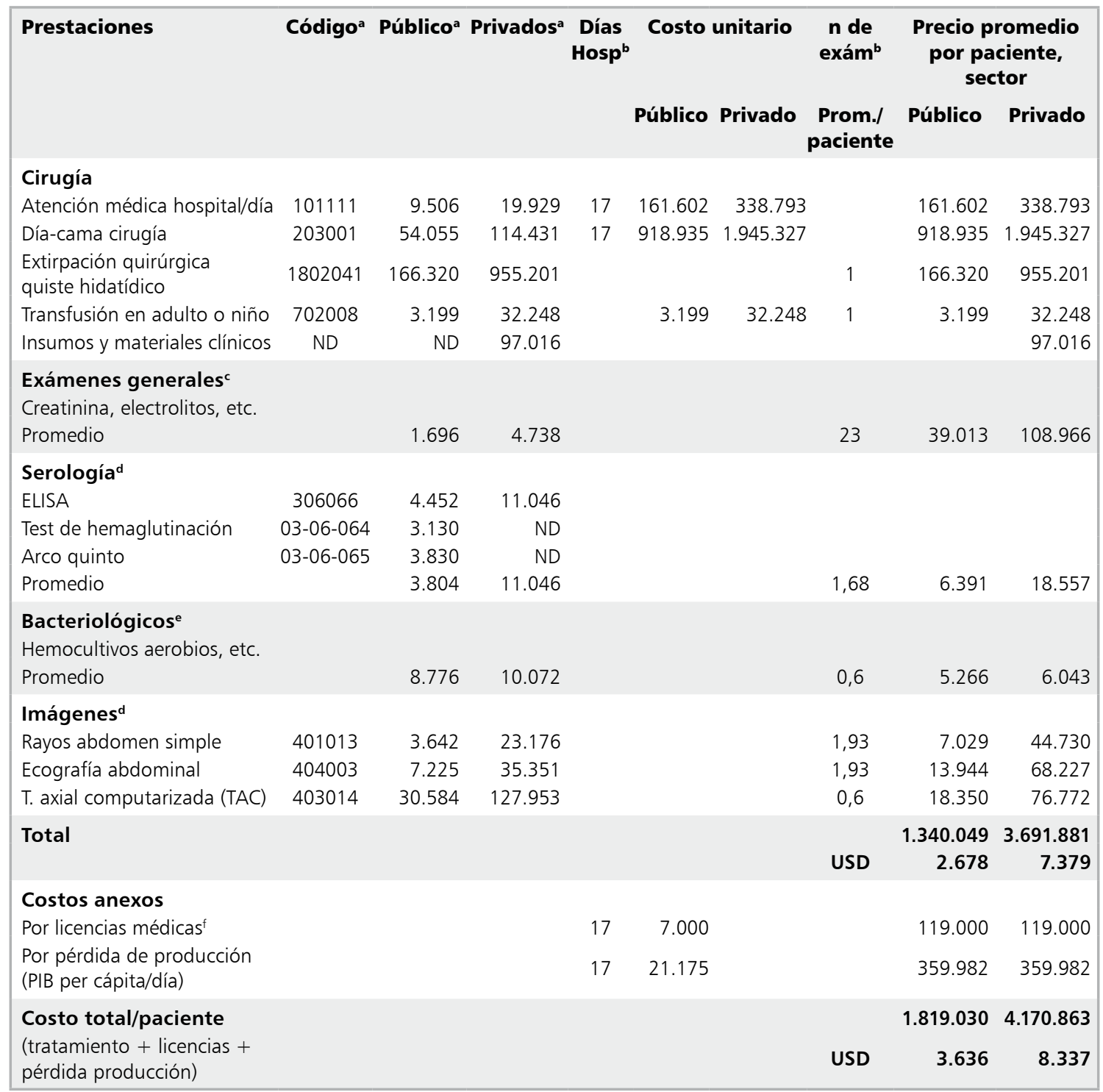

a La gran mayoría de estos datos se recopilaron del Informe Final del Ministerio de Salud (2013) ${ }^{11}$ sobre un estudio de verificación del costo de tratamientos de las enfermedades del plan AUGE. Datos que no estaban en este informe, se obtuvieron del "Libro de Arancel MLE 2012", FONASA ${ }^{14}$ y de un informe de precios de prestaciones médicas otorgadas por el sector privado del año $2012^{15}$. 'De acuerdo a número de días promedios de hospitalización y exámenes promedios descritos en Lorca et al., $2003^{8}$. 'Los exámenes generales considerados, se basaron en Lorca et al., 20038, con sus correspondientes códigos ${ }^{11}$ fueron: Creatinina (302013), electrolitos plasmáticos (302032), gases arteriales (302046), glicemia (302047), grupo y Rh (301034), hematocrito (301036), hemograma (301045), orina completa (309022), proteína C reactiva (305031), perfil hematológico (301045), perfil lipídico (302034), protrombina (301059), pruebas hepáticas (302076), TTPK tromboplastina tiempo parcial (301085) y uremia (302057). dDe acuerdo a los exámenes promedios descritos en Lorca et al., 2003. 'Los exámenes de bacteriología, con sus respectivos códigos de FONASA, precios en sector público y privado ${ }^{11}$ son los siguientes: Hemocultivos aerobio (306009-\$ 14.339-\$10.534), Hemocultivo anaerobio (306010-\$ 8.140-\$14.446), Urocultivos (306011-\$4.253-\$5.102) y Bacilos de Koch (306018-\$ 8.372-\$10.206). fSe estimó de acuerdo al salario mínimo imponible actual de $\$ 210.000$, dividido por 30 días, da \$7.000 que multiplicado por el número de días promedios de hospitalización (17), da la cifra indicada en la Tabla. 
Impacto económico de la hidatidosis en Chile - J. Venegas et al

(\$119.000) y pérdidas en producción (\$359.982), da un costo total por paciente de $\$ 1.819 .030$, equivalente a USD 3.636 (dólar observado al 4 de octubre de 2013 de 500,3) (Tabla 2). El mismo procedimiento, pero en el sector privado, nos da una costo total de USD 8.337.

\section{Estimación de perros infectados a nivel regional y nacional}

Para estimar el número total de perros infectados, se consideraron los datos de prevalencia publicados en diferentes regiones de Chile ${ }^{2,12-21}$, los datos de las poblaciones humanas de cada región ${ }^{22}$ y el número de perros estimados por cada región de acuerdo a la razón nacional de habitantes/ perros $6,8: 1^{1,23}$ (Tabla 3). Basados en estos datos, nos da un número total de perros infectados de 53.885 (Tabla 3).
Hallazgos de quistes hidatídicos en animales de abasto a nivel regional y nacional

En la Tabla 4, se muestran el número de animales decomisados por hallazgos de quistes hidatídicos en sus vísceras en los años 2010 y 2011, en cada región del país ${ }^{24,25-27}$. Se observa que en casi todas las regiones de Chile, se decomisaron vísceras de animales faenados en mataderos. El mayor decomiso se realizó en bovinos (76.068 animales), seguidos por porcinos (32.352), ovinos (13.553), equinos (807), caprinos (405) y camélidos (200).

\section{Estimación del impacto económico de la hidati- dosis en producción animal}

Este impacto se estimó en dos áreas, por pérdidas debidas al decomiso de hígados (Tablas 4 y 5) y por la disminución de la producción de carne de la carcasa (Tabla 6). Basados en que 95\% de los

Tabla 3. Estimación del número de perros infectados con Echinococcus spp. por regiones de Chile

\begin{tabular}{|c|c|c|c|c|}
\hline Región & $\begin{array}{c}\text { n de } \\
\text { habitantes }^{\mathbf{a}}\end{array}$ & $\begin{array}{l}\text { n de perros } \\
\text { estimados }^{b}\end{array}$ & $\begin{array}{l}\text { Prevalencia de } \\
\text { equinococosis canina }\end{array}$ & $\begin{array}{l}\text { n de perros } \\
\text { Infectados }^{d}\end{array}$ \\
\hline XV de Arica y Parinacota & 213.595 & & & \\
\hline I. Tarapacá & 298.257 & 43.861 & ND & \\
\hline Il de Antofagasta & 542.504 & & & \\
\hline III de Atacama & 290.581 & & & \\
\hline IV de Coquimbo & 704.908 & 103.663 & 7,2 & 7.464 \\
\hline V de Valparaíso & 1.723 .547 & & & \\
\hline XIII Metropolitana & 6.683 .852 & & & \\
\hline VI de $O^{\prime}$ Higgins & 872.51 & & & \\
\hline VII del Maule & 963.618 & 141.708 & 11 & 15.588 \\
\hline VIII del Biobío & 1.965 .199 & 288.999 & 3,9 & 11.271 \\
\hline IX de La Araucanía & 907.333 & 133.431 & 13,6 & 18.147 \\
\hline XIV de Los Ríos & 363.887 & 53.512 & ND & \\
\hline X de Los Lagos & 785.169 & 115.466 & ND & \\
\hline XI de Aysén & 98.413 & 14.472 & 6,5 & 941 \\
\hline XII de Magallanes y La Antártica & 159.102 & 23.397 & 1,8 & 421 \\
\hline Total país & 16.572 .475 & & 7,6 & 53.831 \\
\hline
\end{tabular}

aResultados Preliminares Censo de Población y Vivienda 2012. Instituto Nacional de Estadísticas: Período de la Información: 9

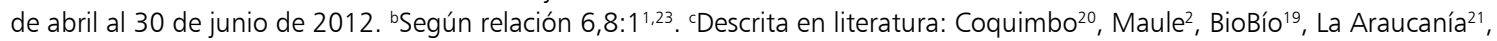
Aysén ${ }^{18}$ y Magallanes ${ }^{17}$. Para estimar la prevalencia "total país", se consideró sólo los datos de las regiones IV, VII, VIII, IX, XI y XII. 'Sólo se consideraron las regiones IV, VII, VIII, IX, XI y XII, en los que había datos de prevalencia. 
Tabla 4. Número total de animales en los cuales se notificó presencia de quistes hidatídicos el año 2010 - 2011, recopilados en informes semestrales del Servicio Agrícola y Ganadero de Chile (SAG)

\begin{tabular}{|c|c|c|c|c|c|c|}
\hline Regiones & Bovinos $^{a}$ & Camélidos ${ }^{\mathrm{a}}$ & Caprinos $^{\mathbf{b}}$ & Equinos $^{b}$ & Ovinos $^{a}$ & Porcinos $^{b}$ \\
\hline XV de Arica y Parinacota & 48 & 200 & 0 & 0 & 13 & 0 \\
\hline I. Tarapacá & 0 & 0 & 0 & 0 & 0 & 0 \\
\hline II de Antofagasta & 0 & 0 & 0 & 0 & 0 & 0 \\
\hline III de Atacama & 0 & 0 & 0 & 0 & 0 & 0 \\
\hline IV de Coquimbo & 2.149 & 0 & 187 & 32 & 414 & 11 \\
\hline V de Valparaíso & 2.120 & 0 & 0 & 53 & 0 & 8 \\
\hline XIII Metropolitana & 17.364 & 0 & 0 & 2 & 13 & 4 \\
\hline VI de O'Higgins & 4.534 & 0 & 0 & 2 & 7 & 16.114 \\
\hline VII del Maule & 5.459 & 0 & 15 & 0 & 87 & 515 \\
\hline VIII del Biobío & 5.769 & 0 & 0 & 7 & 2.994 & 385 \\
\hline IX de La Araucanía & 13.632 & 0 & 203 & 625 & 852 & 2.521 \\
\hline XIV de Los Ríos & 7.475 & 0 & 0 & 0 & 0 & 0 \\
\hline X de Los Lagos & 11.755 & 0 & 0 & 14 & 805 & 12.777 \\
\hline XI de Aysén & 3.423 & 0 & 0 & 72 & 1.244 & 17 \\
\hline XII de Magallanes y La Antártica & 2.340 & 0 & 0 & 0 & 7.124 & 0 \\
\hline Total país & 76.068 & 200 & 405 & 807 & 13.553 & 32.352 \\
\hline
\end{tabular}

${ }^{a}$ Decomisos informados por el SAG año $2011^{25,26}$. becomisos informados por el SAG año $2010^{27}$.

decomisos se produce por quistes en el hígado 28,29 y el peso promedio de cada hígado por especie (Tabla 6), se observó que la mayor pérdida se produce por el decomiso de hígados de bovinos, seguida por porcinos y ovinos, dando un monto total estimado en el año 2011 de USD 1.449.931 (Tabla 6).

El procedimiento para estimar las pérdidas económicas ocasionadas en producción de carne en vara (carcasa muscular sin vísceras), se muestra en la Tabla 7. En base a dichos cálculos, se obtuvo una pérdida total de USD 8,7 millones, siendo las mayores pérdidas en bovinos, seguida por porcinos y ovinos (Tabla 7).

\section{Estimación de pérdidas totales producida por hidatidosis a nivel humano y animal}

La estimación de los costos totales a nivel humano y animal, se muestran en la Tabla 8. Así, el costo producido por el tratamiento quirúrgico de la hidatidosis, se basó en los porcentajes de atención de pacientes en el sector público (81\%) y privado (19\%), informados por el Minsal ${ }^{11}$, correspondiente en nuestro caso a 559 y 131 pacientes, originando un costo total de USD 2,46 millones (Tabla 8). Sumando las licencias médicas y pérdidas en producción, el costo a nivel humano anual que produciría la hidatidosis en Chile sería de USD 3,13 millones (Tabla 8).

Sumando los costos en el área humana y animal, se obtiene un costo total anual de USD 14,35 millones (Tabla 8).

\section{Discusión}

\section{Impacto a nivel humano}

La estimación del costo producido por el tratamiento quirúrgico de la hidatidosis por paciente, informada en el presente artículo, muestra una gran diferencia de precios entre el sector público y privado de (USD 2.678 y USD 7.379, respectivamente. En un estudio similar ${ }^{8}$, realizado el año 2001 en un grupo de pacientes del hospital San Borja de Santiago, se estimó un costo mínimo y máximo de USD \$ 880 y USD \$ 4483, con una 
Impacto económico de la hidatidosis en Chile - J. Venegas et al

Tabla 5. Incidencia de hidatidosis en seres humanos, prevalencia de equinococosis canina y porcentaje de hallazgos de quistes hidatídicos en mataderos de animales de abasto en diferentes regiones de Chile

\begin{tabular}{|c|c|c|c|c|c|c|c|c|}
\hline Regiones & $\begin{array}{r}\text { Hur } \\
\text { ENO }^{6}\end{array}$ & $\begin{array}{l}\text { manos } \\
\text { Egresos }^{4}\end{array}$ & $\begin{array}{c}\text { Perros }^{a} \\
(\%)\end{array}$ & $\begin{array}{c}\text { Bovinos }^{b} \\
(\%)\end{array}$ & $\begin{array}{c}\text { Caprinosc } \\
\text { (\%) }\end{array}$ & $\begin{array}{c}\text { Equinosc } \\
(\%)\end{array}$ & $\begin{array}{c}\text { Porcinos }^{c} \\
(\%)\end{array}$ & $\begin{array}{c}\text { Ovinos }^{\circ} \\
(\%)\end{array}$ \\
\hline XV de Arica y Parinacota & 0,55 & ND & & & ND & & & 0 \\
\hline I. Tarapacá & 0,31 & 1 & ND & & ND & & & 0 \\
\hline II de Antofagasta & 0 & 1 & & & ND & & & 0 \\
\hline III de Atacama & 1,42 & 2 & & & ND & & & 0 \\
\hline IV de Coquimbo & 2,74 & 10,5 & 7,2 & 14,8 & 17,9 & 23,0 & 9,65 & 43,6 \\
\hline V de Valparaíso & 0,23 & 2 & & 7,9 & ND & 0,31 & 8,70 & \\
\hline XIII Metropolitana & 0,24 & 2 & & 14,9 & 0,0 & 0,01 & 0,00 & 0,3 \\
\hline VI de $O^{\prime}$ Higgins & 1,35 & 3,8 & & 32,9 & ND & & 0,60 & 0,4 \\
\hline VII del Maule & 1,48 & 9,8 & 11 & 19,8 & 100 & & 0,27 & 8,5 \\
\hline VIII del Biobío & 3,37 & 9 & 3,9 & 4,8 & 0 & 1,11 & 1,17 & 6,4 \\
\hline IX de La Araucanía & 4,60 & 28,5 & 13,6 & 9,1 & 48,5 & 17,4 & 8,81 & 9,2 \\
\hline XIV de Los Ríos & 5,52 & 9,9 & ND & 12,0 & ND & & & ND \\
\hline X de Los Lagos & 0,59 & ND & ND & 6,8 & ND & & 76,9 & 4,7 \\
\hline XI de Aysén & 35,89 & 44 & 6,5 & 42,9 & 0 & 17,0 & 4,72 & 9,6 \\
\hline $\begin{array}{l}\text { XIl de Magallanes y } \\
\text { La Antártica }\end{array}$ & 2,51 & 16,5 & 1,8 & 32,3 & ND & & 0,00 & 1,0 \\
\hline Total país & 1,48 & 6 & 7,6 & 10,5 & 16,9 & 1,68 & 0,88 & 1,7 \\
\hline
\end{tabular}

${ }^{a}$ Descrita en literatura: Coquimbo ${ }^{20}$, Maule ${ }^{2}$, Biobío ${ }^{19}$, La Araucanía ${ }^{21}$, Aysén ${ }^{18}$ y Magallanes ${ }^{17}$. bPrevalencia estimada en base a datos de sacrificio regionales de cabezas de bovinos el año $2011^{34}$ y decomisos informados por el SAG el año 201125,26. 'Estimada en base a número de cabezas faenadas en cada región el año $2010^{19}$ e informe de decomisos de animales el año $2010^{27}$. 'Estimada en base al número total de cabezas de ovino faenadas el año $2011^{34}$ (791.477) multiplicado por los porcentajes de faenamiento regionales del año $2009^{35}$ y decomisos regionales por causa de hidatidosis el año 201125,26.

Tabla 6. Estimación de pérdidas económicas provocadas por el decomiso de hígados en animales de abastos, año 2011

\begin{tabular}{|c|c|c|c|c|c|}
\hline Especie & $\begin{array}{l}\text { Hallazgos de quistes } \\
\text { (n cabezas) }\end{array}$ & $95 \%$ & $\begin{array}{l}\text { Peso promedioc } \\
\text { hígados }\end{array}$ & Precio/kg ${ }^{d}$ & $\begin{array}{l}\text { Pérdidae } \\
\text { (USD) }\end{array}$ \\
\hline Bovino & 76.068 & 72.264 & 9 & 2 & 1.300 .763 \\
\hline Caprino & 405 & 384,8 & 0,7 & 2 & 538 \\
\hline Equinos & 807 & 766,7 & 5 & 2 & 7.666 \\
\hline Porcinos & 32.352 & 30.734 & 2 & 2 & 12.2937 \\
\hline Ovinos & 13.553 & 12.875 & 0,7 & 2 & 18.025 \\
\hline Total & & & & & 1.449 .931 \\
\hline
\end{tabular}

a Decomisos regionales por causa de hidatidosis (SAG 2011) ${ }^{25,26}$. ${ }^{b}$ Considerando que en $95 \%$ de los animales, los quistes hidatídicos se detectan en el hígado ${ }^{10,18}$. 'En base a datos publicados en: http://mundo pecuario.com/tema226/hígado_vesicula_animales/hígado-1161.html [Consultado el 10 de diciembre de 2012]. 'Estimada en base a datos publicados en ${ }^{29}$. eEstimada de acuerdo al número de cabezas de cada especie (en los cuales se detectó un quiste), multiplicado tanto por el peso como por el precio promedio de cada hígado, se obtiene el costo total producido por decomisos en cada especie animal. 
Tabla 7. Estimación de la pérdida económica producida por la hidatidosis en la producción de carnes en Chile, año 2011

\begin{tabular}{|c|c|c|c|c|c|c|c|c|}
\hline Especies & $\begin{array}{c}\text { Producción } \\
\text { nacional } \\
\text { (Ton.) }^{a}\end{array}$ & $\begin{array}{c}\text { Beneficio } \\
\text { nacional } \\
\text { (Cabezas) }^{a}\end{array}$ & $\begin{array}{c}\text { Carne/ } \\
\text { animal } \\
(\mathbf{k g})^{\mathbf{b}}\end{array}$ & $\begin{array}{l}\text { Decomisos } \\
\text { (Cabezas)' }^{\text {Caber }}\end{array}$ & $\begin{array}{l}\text { Carne prod. } \\
\text { animales con } \\
\text { hidatidosis }{ }^{\mathrm{d}} \\
\text { (Ton.) }\end{array}$ & $\begin{array}{l}\text { Pérdida } \\
\text { (5\%)e } \\
\text { (Ton.) }\end{array}$ & $\begin{array}{l}\text { Precio/ton } \\
\text { (USD) }^{\mathrm{f}}\end{array}$ & $\begin{array}{l}\text { Pérdidas } \\
\text { (USD)g } \\
\text { (miles) }\end{array}$ \\
\hline Bovino & 190.979 & 724.830 & 263,5 & 76.068 & 20.042 & 1.054 & 7.484 & 7.895 \\
\hline Ovinos & 11.176 & 791.477 & 14,1 & 13.553 & 191 & 10,1 & 6.925 & 69,8 \\
\hline Porcinos & 527.857 & 5.272 .128 & 100 & 32.352 & 3.239 & 170 & 3.998 & 681,6 \\
\hline Equinos & 8.324 & 46.454 & 179 & 807 & 145 & 7,6 & 6.833 & 52,0 \\
\hline Caprinos & 18,7 & 1.175 & 15,9 & 405 & 6 & 0,3 & 4.840 & 1,6 \\
\hline Total & & & & & & & & $8.699,6$ \\
\hline
\end{tabular}

${ }^{a}$ Ton. = toneladas. Datos de producción obtenidos de Echavarri et al. (2012) ${ }^{36} \cdot{ }^{b}$ Carne producida en promedio por cada especie de animal. Producción nacional/Beneficio nacional. 'Bovinos y ovinos, datos de decomisos OlE25,26. Caprinos, equinos y porcinos, datos de decomisos OIE (2010) ${ }^{28}$. d(Carne/animal) x decomisos. eSuponiendo pérdida de $5 \%$ del total de la carne producida, de

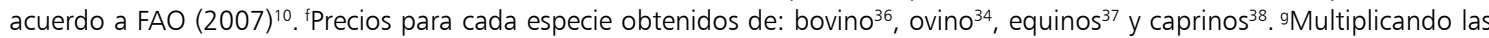
toneladas de carne perdida por los precios respectivos.

Tabla 8. Estimación de los costos totales anuales provocados por la hidatidosis en Chile

\begin{tabular}{|llcccc|}
\hline Hospedero & Tipo de costos & $\begin{array}{c}\text { Costo } \\
\text { unitario } \\
\text { USD }\end{array}$ & $\begin{array}{c}\text { n de } \\
\text { Pacientes }\end{array}$ & $\begin{array}{c}\text { Total de casos } \\
\text { USD }\end{array}$ & $\begin{array}{c}\text { \$c } \\
\text { (millones) }\end{array}$ \\
(millones)
\end{tabular}

a Costo unitario, del tratamiento quirúrgico de la hidatidosis humana, se describe en detalle en la Tabla 2. ${ }^{\text {bEl }}$ total de pacientes sometidos a cirugía, se estimó de acuerdo al número total de casos de notificación obligatoria (ENO) clasificados como CIE 10: B67 notificados por el Minsal para los años $2010^{13}, 2011^{6}$ y $2012^{6}$, el cual da 255,7 multiplicado por 2,7 se obtuvo una cifra de 690 personas sometidas a cirugía. La estimación del número de pacientes atendidos por Fonasa y el sector privado se calculó en base los porcentajes publicados por el Minsal el 2013 ${ }^{11}$, en los cuales se estima que $81 \%$ y $19 \%$ de los pacientes se tratan en el sector público y privado, respectivamente. 'De acuerdo al precio del dólar observado el 4 de octubre de 2013 de $\$$ 500,3 pesos chilenos. 'Tratamiento de perros, se consideró un gasto estimado por animal de USD $20^{10}$. Decomisos de hígados, de acuerdo a costo estimado en Morales (1996) ${ }^{9}$. Las pérdidas en la producción de carne en vara, se estimaron de acuerdo a los diferentes precios publicados de las carnes de cada especie animal (ver Tabla 7). 
mediana de USD 1.465. Debido a que los costos de las prestaciones y exámenes se tomaron de informes de Fonasa, estos costos corresponden al sector público ${ }^{8}$. Comparado con nuestra estimación (USD 2.678), la mediana de USD 1.465 es 1,8 veces inferior. Esta diferencia podría deberse a la inflación acumulada desde el 2001 hasta el 2013 y al alza de precios de las prestaciones, sobre todo las de mayor costo, como la cirugía hepática y los días-camas cirugía. A pesar de esta diferencia, nuestra estimación esta dentro del rango publica$\mathrm{do}^{8}$ del menor y mayor costo, apoyando la certeza de nuestra estimación. En otro estudio semejante publicado por la FAO del $2007^{10}$, se estimó un costo del tratamiento quirúrgico de la hidatidosis en USD 4.440, el cual también está en el rango de costos en el sector público y privado, calculado por nosotros.

Las pérdidas por concepto de licencias médicas, en Lorca et al. ${ }^{8}$ se estimaron en USD 127 y en nuestro caso en USD 238. Diferencia que se debe exclusivamente a los precios del Dólar observado entre el año 2001 (\$702) y el actual (\$500,3 3 octubre de 2013), pues se utilizó el mismo número de días licencia (17 días). En dicho estudio no se estimó la perdida de producción debida a los días no trabajados.

Comparado el costo de tratamiento quirúrgico de la hidatidosis ( $\$ 1,34$ millones en el sector público) con el costo del tratamiento más costoso del grupo de enfermedades $\mathrm{GES}^{11,30}$, el cual corresponde al trasplante renal ( $\$ 13,5$ millones de pesos $)^{11}$, se observa que el primero equivale a 9,9\% del segundo. Esta comparación resalta el gran costo a nivel personal que significa la hidatidosis en nuestro país, destacando que esta estimación corresponde al costo mínimo en el sector público, sin considerar ningún tipo de complicación, costo de tipo psicológico o social. Resaltando además, que en la mayoría de los casos se trata de personas en edad productiva y jefes de hogar.

Haciendo un paralelo entre el costo total anual del tratamiento quirúrgico de la hidatidosis, suponiendo una demanda total de 690 pacientes con sus respectivos porcentajes de atención en el sector público y privado ${ }^{11}$, y comparándolo con la jerarquía de costos en orden decreciente, de las 81 enfermedades $\mathrm{GES}^{11}$, se observa que la hidatidosis quedaría en el lugar 72, entre la hepatitis B (\$1.400 millones) y el tratamiento del consumo de alcohol y drogas en personas menores de 20 años (\$ 1.144 millones). En esta jerarquía de costos, es importante mencionar que otra enfermedad infecciosa, como la neumonía adquirida en personas de 65 años, aparece con un costo total de $\$ 622$ millones.

\section{Impacto de la hidatidosis en producción animal}

Estimaciones previas para cuantificar las pérdidas por decomisos de hígados y riñones en varios animales de abastos arrojaron una cifra de USD $1.607 .328^{9}$, la cual es cercana a la estimada por nosotros de USD 1.449.931 (Tabla 5), sugiriendo que nuestra estimación es correcta.

En el trabajo de Morales $(1996)^{9}$, no se realizaron estimaciones sobre el impacto económico de la hidatidosis en la producción de carnes en Chile. En el estudio de la FAO-RLC $(2007)^{10}$, se analizó esta variable en conjunto con el efecto de decomisos, y otros factores, arrojando un costo estimado entre USD 7 y 11,2 millones. En nuestra estimación, debido a la carencia de datos sólidos, en otros ámbitos de la producción animal, sólo se consideró el efecto de la hidatidosis en los decomisos de hígados y en la producción de carne, dándonos un valor de USD 8.699.614. Resultado que está dentro del rango estimado por el informe de FAO-RLC $(2007)^{10}$, apoyando que nuestra estimación es correcta.

La estimación del costo total que produce la hidatidosis en nuestro país, se obtuvo sumando las pérdidas producidas a nivel humano y animal, lo que nos dio una cifra anual de USD 14,35 millones, cifra que también está en el rango de la estimación publicada por la FAO el $2007^{10}$.

\section{Posibles causas que han dificultado la erradicación de la hidatidosis en Chile}

En el presente trabajo, al comparar las tasas de hallazgos de quistes hidatídicos en animales con las tasas de incidencias de hidatidosis en seres humanos (Tabla 8), se observa que hay una coincidencia entre las altas tasas de ambos grupos. Las tasas en animales representan el grado de contaminación ambiental con huevos del parásito. Por tal razón, dichas tasas coinciden con las altas prevalencias de infección publicadas para perros ${ }^{2,17,18,19,21}$. Destacar que, en las regiones que muestran los mayores índices a nivel humano y animal como son, Aysén, La Araucanía, Coquimbo y Los Ríos, podrían existir diferentes ciclos de transmisión del parásito ${ }^{31-33}$.

Otras causas que pudiera explicar la persis- 
tencia de altos índices a nivel humano y animal, son la subnotificación de esta enfermedad ${ }^{2-5}$, las persistencias de conductas de riesgos de las comunidades locales, deficiente tenencia de los perros, insuficientes campañas educativas-preventivas, etc $^{1,2,33}$. Varias de estas causas, se evidencian en las diferentes Tablas mostradas en el presente artículo. Así por ejemplo, la primera de ellas se observa en las discordancias entre, las tasas notificadas y las tasas de egresos hospitalarios (Tabla 1). Las otras causas, se manifiestan en la mantención casi constante de las tasas de incidencia en humanos por largos período de tiempo (Tabla 1), etc.

Finamente consideramos que el presente trabajo, al estimar el costo económico que produce la hidatidosis en nuestro país, actualiza los análisis acerca de su impacto y entrega nueva información que podría ser muy útil para mejorar las actuales campañas de control y vigilancia epidemiológica de esta enfermedad en nuestro país.

\section{Referencias}

1. Eckert J, Gemmell M.A, Meslin F-X, Pawłowski ZS. WHO/OIE: Manual on Echinococcosis in Humans and Animals: a Public Health Problem of Global Concern. 2001.

2. Apt W, Pérez C, Galdámez E, Campano S, Vega F, Vargas $\mathrm{D}$, et al. Echinococcosis/hidatidosis in the VII region of Chile: diagnosis and educational intervention. Panamerican Journal Public Health 2000; 7: 8-16.

3. Noemí I, Viovy A, Zamorano RP, Blanco AM, Revello DP, Vojkovic ML, et al. Hidatidosis en la infancia: Albendazol en su tratamiento médico y quirúrgico. Rev Chilena Infectol 2003; 20: 229-34.

4. Cortés S, Valle C. Hidatidosis humana: Generalidades y situación epidemiológica en Chile según egresos hospitalarios y notificación obligatoria entre los años 2001 y 2005. Rev Chilena Infectol 2010; 27 (4): 329-35.

5. Martínez P. Hidatidosis humana: antecedentes generales y situación epidemiológica en Chile, 2001-2009. Rev Chilena Infectol 2011; 28 (6): 585-91.

6. Fuenzalida F. Hidatidosis (CIE 10: B67). Situación epidemiológica, semanas 1 A 52, AÑO 2012 (01/01/2012 AL 29/12/2012). Dpto. de Epidemiología, DIPLASMINSAL. Norma Técnica No 55 de Vigilancia de enfermedades. 2012.

7. Ministerio de Salud. Objetivos Sanitarios para Chile 2000-2010. Ministerio de Salud (Minsal), Departamento de Epidemiología, Primera edición, octubre de 2002.
8. Lorca M, Campanella C, Gavrilovics A, Lagos L, Florín C. Estimación de los costos totales y parciales de diagnóstico, tratamiento quirúrgico y seguimiento de la hidatidosis hepática en el Hospital San Juan de Dios, Santiago, Chile 1990-1999. Rev Chil Cir 2003; 55 (1): 60-4.

9. Morales MA. Decomisos y su importancia económica en mataderos de Chile. Tecno Vet 1996; 2 (1): 1-3.

10. FAO. Organización de las Naciones Unidas para la Agricultura y la Alimentación. Oficina Regional para América Latina y el Caribe FAO/RLC. Estimación del impacto económico de la equinococosis quística en el cono sur (Argentina, Brasil, Chile y Uruguay). Junio de 2007; págs 1-20.

11. INE-2012. www.latercera.com/noticia/negocios/2012/ 12/655-497836-9-ine-ingreso-mensual-promedio-delos-chilenos-fue-de-390365-en-2011.shtml. [Consultado el 10 de diciembre de 2012].

12. Organización Panamericana de la Salud (OPS). Clasificación Estadística Internacional de Enfermedades y Problemas relacionados con la Salud. Décima revisión. 1995.

13. Fuentes R. Situación epidemiológica. Semanas 1 a 52 año 2011 (\&). Departamento de Epidemiología. División de Planificación Sanitaria, Ministerio de Salud de Chile. 21 de noviembre de 2012, págs. 1-2.

14. Libro de Arancel MLE 2012, FONASA. Arancel de Prestaciones de Salud. Modalidad Libre Elección.

15. Prestaciones Médicas otorgadas por el sector privado, enero-diciembre de 2012. Cuadro $\mathrm{N}^{\circ}$ 3.7.1. http://www. supersalud.gob.cl/documentacion/569/w3-propertyvalue-3749.html (Consultado el 8 de octubre de 2013).

16. PIB per cápita 2012. Banco Central: http://si3.bcentral. $\mathrm{cl} /$ Siete/secure/cuadros/arboles.aspx (Consultado el 3 de octubre de 2013).

17. Álvarez F, Tamayo R, Ernst S. Estimación de la prevalencia de equinococosis canina en la XII Región, Chile, 2002. Parasitol Latinoam (FLAP) 2005; 60: 74-7.

18. Moro PL, Schantz PM. Echinococcosis: historical landmarks and progress in research and control. Annual of Tropical Medicine of Parasitology 2006; 100: 703-14.

19. Villa CO. Determinación de la prevalencia de equinococosis canina y el grado de conocimiento que la población posee con respecto a la enfermedad, en el área urbana de San Fabián de Alico, provincia de Nuble. Tesis para optar al título de médico veterinario. Universidad de Concepción. http://www.tesischilenas.cl/index.php/ record/view/17793 (Consultado el 26 de noviembre de 2012).

20. Acosta-Jamett G, Cleaveland S, Bronsvoort B, Cun- 
ningham A, Bradshaw, Craig PS. Echinococcus granulosus infection in domestic dogs in urban and rural areas of the Coquimbo region, north-central Chile. Veterinary Parasitology 2010; 169: 117-22.

21. Armstrong WA. Sistema de notificación on-line de Zoonosis. Plan piloto de la SEREMI de Salud Araucanía. Jefe Departamento de Acción Sanitaria. 2012.

22. Resultados Preliminares Censo de Población y Vivienda 2012. Instituto Nacional de Estadísticas: Período de la Información: 9 de abril al 30 junio de 2012.

23. Organización Panamericana de la salud. Resumen de la situación de los programas de rabia en América Latina. Informe final. RIMSA 2003; 13/INF/2 (Sp): 3-63.

24. Informe beneficio y hallazgos patológicos en mataderos nacionales 2010. Subdepartamento de vigilancia epidemiológica división de protección pecuaria. Servicio Agrícola y Ganadero (SAG). Abril de 2011.

25. Mundo Pecuario 2012. http://mundo-pecuario.com/ tema226/higado_vesicula_animales/higado-1161.html [Consultado el 10 de diciembre de 2012].

26. Cancino P. Informe semestral para la notificación de la ausencia o presencia de enfermedades de la lista de la OIE. Referencia OIE: 113308, País: Chile. Período del informe enero-junio de 2011.

27. Cancino P. Informe semestral para la notificación de la ausencia o presencia de enfermedades de la lista de la OIE. Referencia OIE: 120838, País: Chile. Período del informe julio-diciembre de 2011.

28. Informe anual de salud animal para la notificación de la ausencia o presencia de todas las enfermedades Referencia OIE: 100064, 99485, 89771, País: Chile. Período del informe enero-diciembre de 2010.

29. Morales MA, Luengo J. Beneficios y causales del decomiso de ovinos, porcinos, equinos, caprinos y camélidos en Chile. Avances en Ciencias Veterinarias, 1995; 10 (2). págs 1-7.
30. Anexo: Canasta Valoradas. Ministerio de Salud, 11 de febrero de 2013.

31. Nakao M, McManus DP, Schantz PM, Craig PS, Ito A. A molecular phylogeny of the genus Echinococcus inferred from complete mitochondrial genomes. Parasitology 2007; 134: 713-22.

32. Moks E, Jogisalu I, Valdmann H, Saarma U. First report of Echinococcus granulosus genotype G8 in Eurasia and a reappraisal of the phylogenetic relationships of 'genotypes' G5-G10 Parasitology 2008; 135 (5): 647-54.

33. Moro P, Schantz PM. Echinococcosis: a review. International Journal of Infectious Diseases 2009; 13: 125-33.

34. Velis H. Producción Pecuaria años 2006-2011. Publicación Semestral, Instituto Nacional de Estadísticas. Subdirección de Operaciones; Departamento de Estadísticas Agropecuarias; Departamento de Comunicaciones e Imagen Corporativa. Fecha de Publicación: 31 de mayo de 2012.

35. Consorcio Ovino 2010. Indicadores Ovinos No 4. Carne Ovina-Empezando el 2010. Gobierno de Chile, Fundación para la Innovación Agraria. Consorcio Ovino. www. consorcioovino.cl - info@consorcioovino.cl.

36. Echávarri V, López I, Amunátegui R, De la Fuente T. Boletín: Carne bovina: tendencias de producción, precios y comercio exterior. ODEPA, Información a marzo de 2012 para beneficio y producción. Información a abril de 2012 para precios y comercio exterior. Ministerio de Agricultura, mayo de 2012.

37. Catelli JL, Caviglia JF, Tassara ML, Giménez R. Producción de equinos para carne. Revista de Ciencias Agrarias y Tecnología de los Alimentos Vol. 24 -2006.

38. Producción de Carne Caprina en Lonquimay. Proyecto de Innovación en la IX Región de La Araucanía. Fundación para la Innovación Agraria. Ministerio de Agricultura. 2008. 